\title{
Noncommutative spacetime symmetries: Twist versus covariance
}

\author{
J. M. Gracia-Bondía, ${ }^{1}$ Fedele Lizzi, ${ }^{2, *}$ F. Ruiz Ruiz, ${ }^{1, \dagger}$ and Patrizia Vitale ${ }^{2, *}$ \\ ${ }^{1}$ Departamento de Física Teórica I, Universidad Complutense de Madrid, 28040 Madrid, Spain \\ ${ }^{2}$ Dipartimento di Scienze Fisiche, Università di Napoli Federico II, \\ and INFN, Sezione di Napoli, Monte S. Angelo, Via Cintia, 80126 Napoli, Italy
}

(Received 5 May 2006; published 18 July 2006; corrected 18 July 2006)

\begin{abstract}
We prove that the Moyal product is covariant under linear affine spacetime transformations. From the covariance law, by introducing an $(x, \Theta)$-space where the spacetime coordinates and the noncommutativity matrix components are on the same footing, we obtain a noncommutative representation of the affine algebra, its generators being differential operators in $(x, \Theta)$-space. As a particular case, the Weyl Lie algebra is studied and known results for Weyl invariant noncommutative field theories are rederived in a nutshell. We also show that this covariance cannot be extended to spacetime transformations generated by differential operators whose coefficients are polynomials of order larger than 1 . We compare our approach with the twist-deformed enveloping algebra description of spacetime transformations.
\end{abstract}

\section{INTRODUCTION}

It has been clear since the early days of noncommutative field theory that Lorentz invariance, or the lack of it, plays a key role in the subject, especially when it comes to discuss questions like causality or unitarity. In this regard, one ought to remember there are two distinct types of Lorentz transformations [1]. On the one hand, there are observer Lorentz transformations. They involve coordinate changes under which the localized field configurations and background fields, in this case the noncommutative parameter tensor $\Theta=\left(\theta^{\mu \nu}\right)$, transform covariantly, leaving the physics unchanged. On the other hand, there are particle Lorentz transformations. They involve rotations and boosts of only localized fields within a fixed observer frame, leaving $\Theta$ unchanged and hence modifying the physics. On the face of it, the choice of a particular uniform background $\Theta$ breaks Lorentz symmetry to a smaller subgroup [2], namely, the group of transformations that leave $\Theta$ unchanged. This choice is similar to the choice of a vacuum expectation value in "spontaneous symmetry breaking". One should rather not speak here of unbroken/broken symmetry but rather of hidden/manifest invariance, observer invariance accounting for hidden and particle invariance for manifest.

The idea that noncommutative field theory is observer Poincaré invariant has been around for some time [3,4]. In Ref. [4] it was explicitly shown, using functional derivative methods, that noncommutative $U(n)$ gauge theory is observer Weyl invariant. Recall that the Weyl group includes the Poincare group and dilatations. Lately this viewpoint has been recovered [5] using the Hopf dual of the twist deformation of the Poincaré enveloping algebra.

The twist approach to Poincaré spacetime transformations in noncommutative field theory has its origin in

\footnotetext{
*Electronic address: lizzi@na.infn.it

${ }^{\dagger}$ Electronic address: ferruiz@ fis.ucm.es

Electronic address: vitale@ na.infn.it
}

Ref. [6] and was developed in Ref. [7], see also [8]. The authors of ref. [7] argue that, if Poincaré invariance did not survive in noncommutative field theory, Wigner's particle classification in terms of scalar, vector, spinor fields and so on would not be appropriate. An extension to 4dimensional special conformal transformations has also been considered [9]. The same ideas have been used to study the action of the conformal group in two noncommutative spacetime dimensions [10], where there is an infinite number of generators. They have also been used to explore noncommutative formulations of gravity [11] and gauge theories [12].

In this paper we undertake the study of noncommutative spacetime transformations from the observer point of view with new methods. We start by considering linear affine transformations and find a transformation law for $\Theta$ that ensures affine covariance of the Moyal product. From the infinitesimal formulation of this covariance, expressions for the transformation generators as differential operators in an $(x, \Theta)$-space are obtained. These generators by construction satisfy the Leibniz rule for the Moyal product and the same commutation rules as for commutative spacetime. In terms of them the study of symmetries becomes very simple, and the discussion of the invariance of a classical action in field theory for $\Theta=0$ is easily transferred to the case $\Theta \neq 0$. We also consider the generalization of the twist-deformed enveloping algebra approach to diffeomorphisms and give a closed form for the twisted coproduct of an arbitrary infinitesimal generator. This generalization leads to a neat interpretation of the twist description and allows us a clear comparison between the covariant description of hidden symmetries and the twist formalism.

The paper is organized as follows. In Sec. II we show that the Moyal product is globally covariant under linear affine transformations and obtain their infinitesimal generators in $(x, \Theta)$-space. We particularize to Weyl transformations in Sec. III and obtain in $(x, \Theta)$-space their generators $\left\{P_{\mu}^{\Theta}, M_{\mu \nu}^{\Theta}, D^{\Theta}\right\}$. From this, we give a very sim- 
ple and straightforward proof of Weyl invariance for noncommutative $U(n)$ gauge theory. Section IV is dedicated to investigate spacetime transformations whose infinitesimal form is polynomial in the Cartesian coordinates, of which special conformal transformations are an example. We find that the Moyal product is not covariant for them. In Sec. V we thoroughly compare the observer approach with the twisted coproduct approach for the Poincare algebra. Section VI collects our conclusions. We include a short mathematical appendix.

\section{LINEAR AFFINE COVARIANCE OF THE MOYAL PRODUCT}

We find in Subsec. II A the transformation law for $\Theta$ that renders the Moyal product covariant under linear affine transformations of spacetime. In Subsec. II B we exhibit the infinitesimal generators for such transformations.

\section{A. Global analysis}

Given two functions $f$ and $g$ defined on $\mathbb{R}^{4}$ and any $4 \times$ 4 real antisymmetric matrix $\Theta$, Rieffel's formula [13] for the Moyal star product of $f$ with $g$ is

$$
\begin{aligned}
\left(f \star_{\Theta} g\right)(x)= & \frac{1}{(2 \pi)^{4}} \int d^{4} u d^{4} v f\left(x+\frac{1}{2} \Theta u\right) \\
& \times g(x+v) e^{i u \cdot v} .
\end{aligned}
$$

This definition generalizes others in the literature in that it does not require $\Theta$ to be nondegenerate. It is also valid for any number $n$ of dimensions, even or odd, by simply replacing 4 by $n$. With $f=x^{\mu}$ and $g=x^{\nu}$, expression (1) reproduces the commutation relations

$$
\left[x^{\mu}, x^{\nu}\right]_{\star_{\Theta}}:=x^{\mu} \star_{\Theta} x^{\nu}-x^{\nu} \star_{\Theta} x^{\mu}=i \theta^{\mu \nu} .
$$

Formal expansion of $f$ in the integrand in powers of $\Theta$ and integration by parts yields

$$
\left(f \star_{\Theta} g\right)(x)=\left.\exp \left[\frac{1}{2} \theta^{\mu \nu} \partial_{\mu}^{(x)} \partial_{\nu}^{(y)}\right] f(x) g(y)\right|_{y=x},
$$

which is the form for the Moyal product most often quoted in the field theory literature. A rigorous mathematical derivation of a formula of the type of (2) as an asymptotic development of the exact formula can be found in [14]. It is important to note, however, that whereas expansions like (2) are local, expression (1) is not.

Let us now consider the group of linear affine transformations. We recall that a transformation $\Omega=(L, a)$ of this type is characterized by a real $4 \times 4$ matrix $L$ with nonvanishing determinant and a vector $a$ in $\mathbb{R}^{4}$. On a vector $x \in \mathbb{R}^{4}$ it acts by

$$
x \mapsto \Omega \cdot x=L x+a .
$$

The group product is given by $\Omega \Omega^{\prime}=\left(L L^{\prime}, L a^{\prime}+a\right)$ and the inverse of the transformation $\Omega=(L, a)$ is $\Omega^{-1}=$ $\left(L^{-1},-L^{-1} a\right)$. The action of linear affine transformations on functions on $\mathbb{R}^{4}$ is given by

$$
[\Omega \cdot f](x)=f\left(\Omega^{-1} \cdot x\right)=f\left(L^{-1}(x-a)\right) .
$$

With this definition, we have $[\Omega \cdot f](\Omega \cdot x)=f(x)$ and

$$
\Omega_{1} \cdot\left[\Omega_{2} \cdot f\right]=\left(\Omega_{1} \Omega_{2}\right) \cdot f .
$$

Had we taken the action on a function $f$ to be defined by $[\Omega \cdot f](x)=f(\Omega \cdot x)$, we would have obtained $\Omega_{1} \cdot\left[\Omega_{2}\right.$. $f]=\left(\Omega_{2} \Omega_{1}\right) \cdot f$, which looks less natural. Here we stick to (4).

To investigate the covariance of the star product $\star_{\Theta}$ under the linear affine group, we need to compute $[\Omega$. $f] \star_{\Theta}[\Omega \cdot g]$. Using Rieffel's definition, we obtain

$$
\begin{aligned}
\left([\Omega \cdot f] \star_{\Theta}[\Omega \cdot g]\right)(x)= & \frac{1}{(2 \pi)^{4}} \int d^{4} u d^{4} v \\
& \times f\left(\Omega^{-1} \cdot\left(x+\frac{1}{2} \Theta u\right)\right) \\
& \times g\left(\Omega^{-1} \cdot(x+v)\right) e^{i u \cdot v} .
\end{aligned}
$$

Noting that $\Omega^{-1} \cdot\left(x+x_{0}\right)=\Omega^{-1} \cdot x+L^{-1} x_{0}$ and making the changes of variables $u \rightarrow\left(L^{-t}\right) u$, where $L^{-t}:=$ $\left(L^{-1}\right)^{t}=\left(L^{t}\right)^{-1}$ is the contragredient matrix, and $v \rightarrow L v$, we arrive at

$$
\begin{aligned}
\left([\Omega \cdot f] \star_{\Theta}[\Omega \cdot g]\right)(x)= & \frac{1}{(2 \pi)^{4}} \int d^{4} u d^{4} v \\
& \times f\left(\Omega^{-1} \cdot x+\frac{1}{2} L^{-1} \Theta L^{-t} u\right) \\
& \times g\left(\Omega^{-1} \cdot x+v\right) e^{i u \cdot v} .
\end{aligned}
$$

It is then clear that if the action of a linear affine transformations on the space of antisymmetric matrices $\Theta$ is defined as congruence,

$$
\Omega \cdot \Theta=L \Theta L^{t}
$$

one has

$$
\begin{aligned}
& {[\Omega \cdot f] \star_{\Theta}[\Omega \cdot g]=\Omega \cdot\left(f \star_{\Omega^{-1} \Theta} g\right) \quad \text { or }} \\
& {[\Omega \cdot f] \star_{\Omega \cdot \Theta}[\Omega \cdot g]=\Omega \cdot\left(f \star_{\Theta} g\right) .}
\end{aligned}
$$

Identities (3)-(6) constitute the starting point for our analysis. They show that the Moyal product is fully covariant under linear affine transformations, provided the matrix $\Theta$ transforms as in (5). One could say that we have generalized "observer" covariance to linear affine transformations. For transformations $\Omega$ such that $\Omega \cdot \Theta=\Theta$, Eqs. (6) take the form

$$
[\Omega \cdot f] \star_{\Theta}[\Omega \cdot g]=\Omega \cdot\left(f \star_{\Theta} g\right) .
$$

In this case there is no distinction between "observer" and "particle" linear affine transformations.

Let us study in some detail the set of transformations $\Omega$ for which $\Omega \cdot \Theta=\Theta$. Since the only invariant of congruence is the rank, and any real antisymmetric matrix $\Theta$ has rank 4, 2 or 0 , there are only three orbits of the action (5). 
They, respectively, correspond to the generic set of invertible antisymmetric matrices, to the set of noninvertible, nonvanishing antisymmetric matrices, and to the zero matrix. Assume that $\Theta$ is in the rank-4 orbit and consider matrices $L$ such that $L \Theta L^{t}=\Theta$. They form a group, that we may call the little group. Since the dimension of the little group is that of its Lie algebra, to find it suffices to use the exponential form $L=e^{B}$ and require $L \Theta L^{t}=\Theta$ to first order in $B$. This yields the condition $B \Theta=(B \Theta)^{t}$, which in turn gives ten independent entries for $B$. Hence the little group $G_{4}$ of linear affine transformations that leave invariant the rank-4 orbit has dimension 14 , counting the four translational degree of freedom. Similar arguments show that the little group $G_{2}$ of the rank-2 orbit has dimension 15.

Even if the functions $f$ and $g$ do not depend explicitly on $\Theta$, their Moyal product does. On the basis of this fact, and in accordance with the spirit of covariance, it is convenient to consider an $(x, \Theta)$-space on which a linear affine transformation acts as

$$
\Omega \cdot(x, \Theta)=(L, a) \cdot(x, \Theta)=\left(L x+a, L \Theta L^{t}\right) .
$$

We emphasize that the coordinates which parametrize the variables $x$ and $\Theta$ both change under such a transformation.

\section{B. Infinitesimal generators}

The action (8) possesses infinitesimal generators, which we will generically denote $G^{\Theta}$ and which are vector fields in $(x, \Theta)$-space. As convenient coordinates to express them, we may choose the spacetime coordinates $x^{\mu}$ and six independent entries $\theta^{\mu \nu}$ of $\Theta$. We have for small $B$

$$
\Omega \cdot(x, \Theta)=\left(x+B x+a, \Theta+B \Theta+\Theta B^{t}\right)+O\left(B^{2}\right) .
$$

From this we read the generators, when acting on functions, as

$$
G^{\Theta}:=-\left(a^{\alpha}+B^{\alpha}{ }_{\beta} x^{\beta}\right) \frac{\partial}{\partial x^{\alpha}}-\frac{1}{2}\left(B^{\alpha}{ }_{\gamma} \theta^{\gamma \beta}+\theta^{\alpha \gamma} B_{\gamma}^{\beta}\right) \frac{\partial}{\partial \theta^{\alpha \beta}},
$$

where we have put $B=\left(B^{\alpha}{ }_{\beta}\right)$. The factor $\frac{1}{2}$ in front of the last parenthesis arises because we have chosen as coordinates for $\Theta$ its entries with, say, $\alpha<\beta$ and in (9) we are summing over all $\alpha, \beta$. We may recast (9) as

$$
G^{\Theta}=G_{a}^{\Theta}+G_{B}^{\Theta},
$$

where

$$
G_{a}^{\Theta}=-a^{\alpha} \partial_{\alpha} \quad G_{B}^{\Theta}=-\varepsilon^{\alpha} \partial_{\alpha}+\frac{1}{2} \delta_{\varepsilon} \theta^{\alpha \beta} \frac{\partial}{\partial \theta^{\alpha \beta}} .
$$

Here $\varepsilon(x)$ is the vector field with components $\varepsilon^{\alpha}(x)=$ $B^{\alpha}{ }_{\beta} x^{\beta}$, and

$$
\delta_{\varepsilon} \theta^{\alpha \beta}=-\left(B^{\alpha}{ }_{\gamma} \theta^{\gamma \beta}+\theta^{\alpha \gamma} B_{\gamma}^{\beta}\right)
$$

are the components of the Lie derivative with respect to $\varepsilon(x)$ of the 2-tensor $\Theta=\theta^{\alpha \beta} \partial_{\alpha} \otimes \partial_{\beta}$. We have chosen not to distinguish the matrix $\Theta$ and the corresponding tensor in the notation. We recall in this regard that the Lie derivative with respect to a vector field $v(x)=v^{\gamma}(x) \partial_{\gamma}$ of a contravariant 2-tensor with components $t^{\rho \sigma}$ is given by

$$
\delta_{v} t^{\alpha \beta}=v^{\gamma} \partial_{\gamma} t^{\alpha \beta}-t^{\gamma \beta} \partial_{\gamma} v^{\alpha}-t^{\alpha \gamma} \partial_{\gamma} v^{\beta},
$$

which reduces to (12) for $v^{\alpha}=\varepsilon^{\alpha}$ and $t^{\alpha \beta}=\theta^{\alpha \beta}$ independent of $x$. In $G_{a}^{\Theta}$ we recognize the generators of translations. The term $-\varepsilon^{\alpha} \partial_{\alpha}$ in $G_{B}^{\Theta}$ generates arbitrary linear spacetime transformations. Finally, the term $\frac{1}{2} \delta_{\varepsilon} \theta^{\alpha \beta} \partial / \partial \theta^{\alpha \beta}$ accounts for the linear transformations in the $\Theta$-directions that ensure covariance. The action of the operator $G^{\Theta}$ on the Moyal product $f \star_{\Theta} g$ directly follows from the covariance law (6). Indeed, the infinitesimal version of the latter simply states that

$$
G^{\Theta}\left(f \star_{\Theta} g\right)=G^{\Theta} f \star_{\Theta} g+f \star_{\Theta} G^{\Theta} g .
$$

Hence the generators $G^{\Theta}$ do satisfy the Leibniz rule for the Moyal product of functions. In mathematical terms, the $G^{\Theta}$ are derivations of the Moyal algebra. Note that our way to proceed, i.e., descending from global to infinitesimal covariance, identifies the generators and establishes that they are derivations all at once.

It remains to make explicit the action of each of the generators in (11) on the Moyal product. To do this, we use again Rieffel's formula (1) and obtain, after some algebra,

$$
\begin{array}{r}
\partial_{\alpha}\left(f \star_{\Theta} g\right)=\partial_{\alpha} f \star_{\Theta} g+f \star_{\Theta} \partial_{\alpha} g \\
x^{\alpha}\left(f \star_{\Theta} g\right)=x^{\alpha} f \star_{\Theta} g-\frac{i}{2} \theta^{\alpha \beta} f \star_{\Theta} \partial_{\beta} g \\
=f \star_{\Theta} x^{\alpha} g+\frac{i}{2} \theta^{\alpha \beta} \partial_{\beta} f \star_{\Theta} g
\end{array}
$$

$$
\begin{aligned}
\frac{\partial}{\partial \theta^{\alpha \beta}}\left(f \star_{\Theta} g\right)= & \frac{\partial f}{\partial \theta^{\alpha \beta}} \star_{\Theta} g+f \star_{\Theta} \frac{\partial g}{\partial \theta^{\alpha \beta}} \\
& +\frac{i}{2}\left(\partial_{\alpha} f \star_{\Theta} \partial_{\beta} g-\partial_{\beta} f \star_{\Theta} \partial_{\alpha} g\right) .
\end{aligned}
$$

Equations (14) and (15) together imply that

$$
\begin{aligned}
\varepsilon^{\alpha} \partial_{\alpha}\left(f \star_{\Theta} g\right)= & \varepsilon^{\alpha} \partial_{\alpha} f \star_{\Theta} g+f \star_{\Theta} \varepsilon^{\alpha} \partial_{\alpha} g \\
& +\frac{i}{2} \delta \theta_{\varepsilon}^{\alpha \beta} \partial_{\alpha} f \star_{\Theta} \partial_{\beta} g .
\end{aligned}
$$

These calculations serve as a check of our argument. Indeed, it is straightforward to see from Eq. (11) that Eqs. (16) and (17) imply the Leibniz rule (13). Particularly, for $f$ and $g$ independent of $\Theta$, one has the elegant

$$
-G_{B}^{\Theta}\left(f \star_{\Theta} g\right)=\varepsilon f \star_{\Theta} g+f \star_{\Theta} \varepsilon g,
$$

where still $\varepsilon=B_{\beta}^{\alpha} x^{\beta} \partial_{\alpha}$. The previous formulae are the heart of the paper, and many things in it flow from them. 
In summary, the generators of translations are derivations, and the generators of linear affine transformations can be made into derivations if such transformations are accompanied by suitable transformations in the $\Theta$-directions. Equation (13) tell us that vector fields with components of degree up to one in the coordinates $x^{\mu}$ can still be regarded as generating symmetries of the Moyal algebra. For this, the linearity in the coordinates $x^{\mu}$ of the components of the vector field $\varepsilon(x)$ is decisive. In Section IV we show that matters are different for higher order polynomial dependence. Note also that, for the simpler case of $\mathbb{R}^{2}$, the matrix $\Theta$ has components $\theta^{\alpha \beta}=\theta \epsilon^{\alpha \beta}$. If in addition $B^{\alpha}{ }_{\beta}=-\delta^{\alpha}{ }_{\beta}$ - the sole nontrivial possibility-we recover the derivation $G=x \cdot \partial+2 \theta \frac{\partial}{\partial \theta}$, already obtained in [15]. From this point of view, our results can be seen as an expansion of the scenario of that paper. See the Appendix for further discussion of this point.

\section{THE WEYL GROUP ON $(x, \Theta)$-SPACE AND ITS ACTION ON FIELDS}

So far nothing we have said involves a choice of metric, nor of an action. Approaches to physical problems based on the affine group are known, the metric-affine theory of gravity [16] among them. However, conventional physical theories require the choice of a metric. Since we are ultimately interested in field theory on Minkowski spacetime, let us adopt the Minkowskian metric. Everything works much the same for the Euclidean metric. Furthermore, we consider the Weyl group $W$ of translations, Lorentz transformations and dilatations. It is a subgroup of the linear affine group and hence the results of the last section apply. According to them, the generators of Lorentz transformations and dilatations in $x$-space receive contributions involving derivatives with respect to $\theta^{\mu \nu}$. In what follows we describe the algebra of these generators.

\section{A. The Weyl algebra in $(x, \Theta)$-space}

In the presence of a metric we are allowed to lower and raise the indices of the different tensors. If $L$ in Eq. (8) accounts for a Lorentz transformation $\delta x^{\alpha}=\omega^{\alpha \beta} x_{\beta}-$ $\omega^{\beta \alpha} x_{\beta}$, we can take for the matrix $B$ in Eq. (9) any of the matrices $M_{\mu \nu}$ with entries $\left(M_{\mu \nu}\right)_{\beta}^{\alpha}=\delta^{\alpha}{ }_{\mu} g_{\nu \beta}-$ $\delta^{\alpha}{ }_{\nu} g_{\mu \beta}$. Substituting in formula (11), we obtain for the derivations in $(x, \Theta)$-space associated to Lorentz transformations

$$
M_{\mu \nu}^{\Theta}=x_{\mu} \partial_{\nu}-x_{\nu} \partial_{\mu}+\theta^{\rho}{ }_{\mu} \frac{\partial}{\partial \theta^{\rho \nu}}-\theta^{\rho}{ }_{\nu} \frac{\partial}{\partial \theta^{\rho \mu}} .
$$

Analogously, if $L$ accounts for a dilatation $\delta x^{\mu}=\lambda x^{\mu}$, formula (12) yields $\delta \theta^{\mu \nu}=-2 \lambda \theta^{\mu \nu}$ and for the the corresponding derivation we have

$$
D^{\Theta}=-x \cdot \partial-\theta^{\mu \nu} \frac{\partial}{\partial \theta^{\mu \nu}} .
$$

By construction, the operators $M_{\mu \nu}^{\Theta}, D^{\Theta}$ and the generators $P_{\mu}^{\Theta}=-\partial_{\mu}$ of translations are derivations of the Moyal star product. It is easy to check that they satisfy the same commutation relations as the generators of the ordinary Weyl Lie algebra, namely

$$
\begin{gathered}
{\left[P_{\mu}^{\Theta}, P_{\nu}^{\Theta}\right]=0 \quad\left[P_{\mu}^{\Theta}, M_{\nu \rho}^{\Theta}\right]=g_{\mu \nu} P_{\rho}^{\Theta}-g_{\mu \rho} P_{\nu}^{\Theta}} \\
{\left[D^{\Theta}, P_{\mu}^{\Theta}\right]=P_{\mu}^{\Theta} \quad\left[D^{\Theta}, M_{\mu \nu}^{\Theta}\right]=0} \\
{\left[M_{\mu \nu}^{\Theta}, M_{\rho \sigma}^{\Theta}\right]=g_{\nu \rho} M_{\mu \sigma}^{\Theta}+g_{\mu \sigma} M_{\nu \rho}^{\Theta}-g_{\mu \rho} M_{\nu \sigma}^{\Theta}-g_{\nu \sigma} M_{\mu \rho}^{\Theta} .}
\end{gathered}
$$

We conclude that $\left\{P_{\mu}^{\Theta}, M_{\mu \nu}^{\Theta}, D^{\Theta}\right\}$ represent the Weyl Lie algebra in $(x, \Theta)$-space.

\section{B. Weyl invariant actions}

In field theory we are interested in fields. We expect them to transform according to irreducible representations of the Poincaré Lie algebra. Let us consider, for example, a local $U(1)$-` gauge field $A_{\mu}(x)$, with classical action

$$
S[A]=-\frac{1}{4 g^{2}} \int d^{4} x F_{\mu \nu}^{\Theta}(x) \star F^{\Theta \mu \nu}(x),
$$

where

$$
F_{\mu \nu}^{\Theta}=\partial_{\mu} A_{\nu}-\partial_{\nu} A_{\mu}-i\left(A_{\mu} \star_{\Theta} A_{\nu}-A_{\nu} \star_{\Theta} A_{\mu}\right)
$$

denotes the noncommutative field strength. We recall that this action is invariant under noncommutative $U(1)$ local gauge transformations, whose infinitesimal form is $\delta A_{\mu}=$ $\partial_{\mu} \lambda-i\left(A_{\mu} \star_{\Theta} \lambda-\lambda \star_{\Theta} A_{\mu}\right)$.

Since $A_{\mu}(x)$ does not depend on $\Theta$, its variation under an infinitesimal transformation

$$
\delta A_{\alpha}(x)=\left[\Omega \cdot A_{\alpha}\right](x)-A_{\alpha}(x),
$$

which in field theory is written as $\delta A_{\alpha}(x)=A_{\alpha}^{\prime}(x)-$ $A_{\alpha}(x)$, is the same as for $\Theta=0$. This means that the action of the generators $\left\{P_{\mu}^{\Theta}, M_{\mu \nu}^{\Theta}, D^{\Theta}\right\}$ on $A_{\alpha}(x)$ is the usual one,

$$
\begin{aligned}
P_{\mu}^{\Theta}\left[A_{\alpha}\right] & =-\partial_{\mu} A_{\alpha} \\
M_{\mu \nu}^{\Theta}\left[A_{\alpha}\right] & =\left(x_{\mu} \partial_{\nu}-x_{\nu} \partial_{\mu}\right) A_{\alpha}+g_{\mu \alpha} A_{\nu}-g_{\alpha \nu} A_{\mu} \\
D^{\Theta}\left[A_{\alpha}\right] & =-(1+x \cdot \partial) A_{\alpha} .
\end{aligned}
$$

The key point now is that, since $P_{\mu}^{\Theta}, M_{\mu \nu}^{\Theta}, D^{\Theta}$ are derivations for the Moyal star product, their action on the field strength $F_{\mu \nu}^{\Theta}$ is functionally the same for all $\Theta$, and, in particular, equal to that for $\Theta=0$. This automatically leads to

$$
\begin{aligned}
P_{\alpha}^{\Theta}\left[F_{\mu \nu}^{\Theta}\right]= & \partial_{\alpha} F_{\mu \nu}^{\Theta} \\
M_{\alpha \beta}^{\Theta}\left[F_{\mu \nu}^{\Theta}\right]= & x_{\alpha} \partial_{\beta} F_{\mu \nu}^{\Theta}-x_{\beta} \partial_{\alpha} F_{\mu \nu}^{\Theta}+g_{\mu \alpha} F_{\beta \nu}^{\Theta}-g_{\mu \beta} F_{\alpha \nu}^{\Theta} \\
& +g_{\nu \alpha} F_{\beta \mu}^{\Theta}-g_{\nu \beta} F_{\alpha \mu}^{\Theta} \\
D^{\Theta}\left[F_{\mu \nu}^{\Theta}\right]= & -(2+x \cdot \partial) F_{\mu \nu}^{\Theta} .
\end{aligned}
$$

The proof of Weyl invariance of $S[A]$ then goes as in the commutative case. We thus recover, without recourse to 
functional derivatives, the results of Ref. [4]. With obvious changes, the arguments above apply to noncommutative $U(n)$ gauge fields. In conclusion, covariance of the Moyal product, hence the knowledge of generators which are derivations of the Moyal product, simplifies the proof of invariance of a field theory action.

\section{NON-AFFINE SPACETIME TRANSFORMATIONS}

The question that naturally arises is whether covariance can be extended to spacetime transformation groups whose generators have coefficients with arbitrary polynomial de- pendence on the coordinates $x^{\mu}$. We answer this question in the negative.

Let us consider a spacetime transformation $x \rightarrow \Omega \cdot x$ whose infinitesimal form is quadratic in $x$. It is generated by linear combinations of differential operators of the form $x_{\mu} x_{\nu} \partial_{\rho}$. For the Moyal product to be covariant under such transformation, the matrix $\Theta$ must transform in such a way that the infinitesimal form (13) of covariance holds. This requires the existence of sets of generators, say $\left\{G_{\mu \nu \rho}^{\Theta}\right\}$, defined as differential operators in $(x, \Theta)$-space which act on the Moyal product as derivations and reduce to $\left\{x_{\mu} x_{\nu} \partial_{\rho}\right\}$ for $\Theta=0$. Now, acting with $x_{\mu} x_{\nu} \partial_{\rho}$ on $f \star_{\Theta} g$ and using Eqs. (15) and (16), we obtain

$$
\begin{aligned}
x_{\mu} x_{\nu} \partial_{\rho}\left(f \star_{\Theta} g\right)= & x_{\mu} x_{\nu} \partial_{\rho} f \star_{\Theta} g+f \star_{\Theta} x_{\mu} x_{\nu} \partial_{\rho} g-\left(\theta^{\alpha}{ }_{\mu} x_{\nu}+\theta^{\alpha}{ }_{\nu} x_{\mu}\right)\left[\frac{\partial}{\partial \theta^{\alpha \rho}}\left(f \star_{\Theta} g\right)-\frac{\partial f}{\partial \theta^{\alpha \rho}} \star_{\Theta} g-f \star_{\Theta} \frac{\partial g}{\partial \theta^{\alpha \rho}}\right] \\
& +\frac{1}{4} \theta^{\alpha}{ }_{\mu} \theta_{\nu}^{\beta}\left(\partial_{\rho} f \star_{\Theta} \partial_{\alpha} \partial_{\beta} g+\partial_{\alpha} \partial_{\beta} f \star_{\Theta} \partial_{\rho} g\right) .
\end{aligned}
$$

It follows that the operator

$$
G_{\mu \nu \rho}^{\Theta}=x_{\mu} x_{\nu} \partial_{\rho}+\left(\theta^{\alpha}{ }_{\mu} x_{\nu}+\theta^{\alpha}{ }_{\nu} x_{\mu}\right) \frac{\partial}{\partial \theta^{\alpha \rho}}
$$

satisfies

$$
\begin{aligned}
G_{\mu \nu \rho}^{\Theta}\left(f \star_{\Theta} g\right)= & G_{\mu \nu \rho}^{\Theta} f \star_{\Theta} g+f \star_{\Theta} G_{\mu \nu \rho}^{\Theta} g \\
& +\frac{1}{4} \theta^{\mu \alpha} \theta^{\nu \beta}\left(\partial_{\rho} f \star_{\Theta} \partial_{\alpha} \partial_{\beta} g+\partial_{\alpha} \partial_{\beta} f \star_{\Theta} \partial_{\rho} g\right) .
\end{aligned}
$$

The last two terms in this equation, containing three partial derivatives, cannot be recast as a derivation for $\star_{\Theta}$ and thus prevent the operator $G_{\mu \nu \rho}^{\Theta}$ from being a derivation. Therefore we do not see a way for the operators $x_{\mu} x_{\nu} \partial_{\rho}$ to be made into derivations in $(x, \Theta)$-space so that the spacetime transformations that they generate become a covariance of the Moyal product.

In particular, special conformal transformations cannot become a covariance of the Moyal product, since they are generated by vector fields $K_{\mu}=x^{2} \partial_{\mu}-2 x_{\mu} x \cdot \partial$, which are particular linear combinations of $x_{\mu} x_{\nu} \partial_{\rho}$. The same clearly holds for vector fields with higher order polynomial dependence on $x^{\mu}$.

\section{COMPARISON WITH THE TWIST-DEFORMED ENVELOPING ALGEBRA FORMALISM}

Our aim here is to understand the twist-deformed description of spacetime transformations for the Moyal product in the light of our approach. We start by observing that the twist deformation of the Poincare enveloping algebra used in [7] to describe noncommutative spacetime and its transformations is a particular instance of a well known procedure to "twist" Hopf algebras, originally due to
Drinfeld. See Ref. [17] for a review and details. In what follows we briefly recall it. If $H$ is a Hopf algebra, denote by id the identity map of $H$ onto itself, by $\Delta$ the coproduct map, and by $\eta$ the counit map from the Hopf algebra to the scalars. Consider an invertible element $\chi$ in $H \otimes H$ that satisfies the conditions

$$
\begin{aligned}
(1 \otimes \chi)(\mathrm{id} \otimes \Delta) \chi & =(\chi \otimes 1)(\Delta \otimes \mathrm{id}) \chi \\
(\eta \otimes \mathrm{id}) \chi & =(\mathrm{id} \otimes \eta) \chi=1 .
\end{aligned}
$$

The element $\chi$ is said to be a counital 2-cocycle for $H$. For such a $\chi$, the twist $\Delta_{\chi}(h)=\chi \Delta(h) \chi^{-1}$, with $h$ in $H$, does define a new coproduct in $H$. The algebra underlying $H$ endowed with the new coproduct $\Delta_{\chi}$ is still a Hopf algebra, called twisted Hopf algebra, which may be denoted by $H_{\chi}$. As Hopf algebras, $H_{\chi}$ and $H$ are isomorphic if $\chi$ has the trivial form $\chi=(\gamma \otimes \gamma) \Delta \gamma^{-1}$, with $\gamma$ an invertible element in $H$ satisfying $\eta(\gamma)=1$. Assume moreover that $H$ has a representation in an associative algebra $\mathcal{F}$ with product $m$. That is, for $h$ in $H$ and $a, b$ in $\mathcal{F}$ one has

$$
m(a \otimes b)=a b
$$

$$
h \cdot(a b)=h \cdot m(a \otimes b)=m(\Delta(h) \cdot(a \otimes b)),
$$

where $m(a, b)$ denotes the product of $a$ and $b$ in $\mathcal{F}$. The twisting of $\Delta$ introduces in $\mathcal{F}$ a twisted product $m_{\chi}$ defined by

$$
m_{\chi}(a \otimes b)=m\left(\chi^{-1} \cdot(a \otimes b)\right)
$$

$H_{\chi}$ is represented in the new algebra by its action through $\Delta_{\chi}(h)$, since 


$$
\begin{aligned}
h \cdot m_{\chi}(a \otimes b) & =h \cdot m\left(\chi^{-1} \cdot(a \otimes b)\right) \\
& =m\left(\Delta(h) \chi^{-1} \cdot(a \otimes b)\right) \\
& =m\left(\chi^{-1} \Delta_{\chi}(h) \cdot(a \otimes b)\right) \\
& =m_{\chi}\left(\Delta_{\chi}(h) \cdot(a \otimes b)\right) .
\end{aligned}
$$

Here we have used, in this order, Eqs. (28) and (27), the definition of $\Delta_{\chi}$ and Eq. (28) again. This equation will play a central part below in understanding how the Moyal product behaves under general spacetime transformations. Furthermore, it is easy to see that the first condition (25) implies that the new twisted product $m_{\chi}$ is associative.

Now, let us consider the Lie algebra $\mathfrak{D}$ of diffeomorphisms, whose generators are vector fields with polynomial coefficients on $\mathbb{R}^{4}$. As Hopf algebra $H$ we take the enveloping algebra $\mathcal{U}(\mathfrak{D})$. Likewise the enveloping algebra of any Lie algebra, the coproduct $\Delta$ is first defined for elements $h$ of $\mathfrak{D}$ by $\Delta(h)=1 \otimes h+h \otimes 1$, and then multiplicatively extended to all of $\mathcal{U}(\mathfrak{D})$ by means of $\Delta\left(h h^{\prime}\right)=\Delta(h) \Delta\left(h^{\prime}\right)$. For the algebra $\mathcal{F}$ carrying a representation of $\mathcal{U}(\mathfrak{D})$, take the algebra of functions on spacetime with the ordinary multiplication $m(f \otimes g)=f g$. Finally, for $\chi$, we take the exponent of the Poisson tensor $\chi_{\Theta}=\exp \left(-\frac{i}{2} \theta^{\mu \nu} \partial_{\mu} \otimes \partial_{\nu}\right)$. This $\chi_{\Theta}$ is clearly in $\mathcal{U}(\mathfrak{D}) \otimes$ $\mathcal{U}(\mathfrak{D})$, has an inverse

$$
\chi_{\Theta}^{-1}=\exp \left(\frac{i}{2} \theta^{\mu \nu} \partial_{\mu} \otimes \partial_{\nu}\right)
$$

and satisfies the cocycle condition nontrivially. The Moyal product under the asymptotic guise (2) is then recovered as the "twisted" product

$$
m_{\chi_{\Theta}}(f \otimes g)=m\left(\chi_{\Theta}^{-1} \cdot(f \otimes g)\right)=f \star_{\Theta} g .
$$

In view of (29), it is clear that the action of a generator $h$ on the Moyal product is determined by $\Delta_{\chi_{\Theta}}(h)$, and conversely. In the sequel, for simplicity of notation, we write
$\Delta_{\Theta}$ for $\Delta_{\chi_{\Theta}}$ and $m_{\Theta}$ for $m_{\chi_{\Theta}}$. For the generators of translations, Lorentz transformations [7] and dilatations [9] the following expressions were obtained, in our notation,

$$
\begin{aligned}
\Delta_{\Theta}\left(P_{\mu}\right)= & P_{\mu} \otimes 1+1 \otimes P_{\mu} \\
\Delta_{\Theta}\left(M_{\mu \nu}\right)= & M_{\mu \nu} \otimes 1+1 \otimes M_{\mu \nu}+\frac{i}{2} \theta^{\alpha \beta}\left[\left(g_{\mu \alpha} P_{\nu}-g_{\nu \alpha} P_{\mu}\right)\right. \\
& \left.\otimes P_{\beta}+P_{\alpha} \otimes\left(g_{\mu \beta} P_{\nu}-g_{\nu \beta} P_{\mu}\right)\right] \\
\Delta_{\Theta}(D)= & D \otimes 1+1 \otimes D-i \theta^{\mu \nu} P_{\mu} \otimes P_{\nu} .
\end{aligned}
$$

These equations precisely show that $\Delta_{\Theta}\left(M_{\mu \nu}\right)$ and $\Delta_{\Theta}(D)$ are not derivations of the Moyal product. From Eq. (31) it was concluded that the Poincare group remains relevant in noncommutative field theory. Whereas the argument is suggestive, it does not directly concern Poincaré invariance. Note that Eq. (29) reflects a rather general geometrical fact, since it places no restriction on the generator $h$ except that of being an infinitesimal diffeomorphism. (The limitation to polynomial coefficients in $h$ arises because formula (2) can only deal with diffeomorphisms of this type.) This is why the generators $K_{\mu}$ of special conformal transformation could be added to the list of computed $\Delta_{\Theta}(h)$ [9]. Now, because we are in the enveloping algebra, Eq. (29) applies to differential operators of any order. The method is thus a recipe to encode the action of arbitrary differential operators with polynomial coefficients on Moyal products.

The previous remark leads in a systematic and simple way to compute the twisted coproduct of the generator of any spacetime transformation. Let us take an infinitesimal spacetime transformation generated by differential operators of the form $x^{\mu_{1}} \cdots x^{\mu_{N}} \partial_{\nu}$. Using Eqs. (14) and (15) to compute its action on $f \star_{\Theta} g$, and invoking the definition of the twisted product $m_{\Theta}$ in (28) and its covariance property (29), it follows that

$$
\begin{aligned}
& \Delta_{\Theta}\left(x^{\mu_{1}} \cdots x^{\mu_{N}} \partial_{\nu}\right)=x^{\mu_{1}} \cdots x^{\mu_{N}} \partial_{\nu} \otimes 1+1 \otimes x^{\mu_{1}} \cdots x^{\mu_{N}} \partial_{\nu}+\sum_{k=1}^{N}\left(\frac{i}{2}\right)_{N \geq c_{k}>\cdots>c_{1} \geq 1}^{k} \theta^{\mu_{c_{1}} \alpha_{c_{1}}} \cdots \theta^{\mu_{c_{k}} \alpha_{c_{k}}\left[\partial_{\alpha_{c_{1}}} \cdots \partial_{\alpha_{c_{k}}}\right.} \\
& \otimes x^{\mu_{1}} \ldots{\stackrel{c}{c_{1}}} \ldots c^{c_{k}} \ldots x^{\mu_{N}} \partial_{\nu}+(-1)^{k} x^{\mu_{1}} \ldots \underbrace{c_{1}} \ldots \underbrace{c_{k}} \cdots x^{\mu_{N}} \partial_{\nu} \otimes \partial_{\alpha_{c_{1}}} \cdots \partial_{\alpha_{c_{k}}}] .
\end{aligned}
$$

Here ${ }^{c_{1}}$ indicates that in the product $x^{\mu_{1}} \cdots x^{\mu_{N}}$ the factor $x^{\mu_{c_{1}}}$ is removed. An equivalent formula can be found in Ref. [11]. Equations (31) follow from a stroke from Eq. (32). Moreover, it is straightforward to verify that

$$
m_{\Theta}\left(\Delta_{\Theta}\left(x^{\mu_{1}} \cdots x^{\mu_{N}} \partial_{\nu}\right) \cdot\left(x^{\alpha} \otimes x^{\beta}-x^{\beta} \otimes x^{\alpha}\right)\right)=0 .
$$

In other words, $\theta^{\alpha \beta}$ remains unchanged. The twisted coproduct formulation accounts only for particle transformations, for transformations of $\Theta$ are left out. To understand observer transformations, one has to look elsewhere, to the analysis presented in this paper.
To summarize our comparison, for $G$ in the affine group the relation between the covariant and twist approaches can be accounted by the following equation

$$
\begin{aligned}
m_{\Theta}\left(\Delta_{\Theta}(G) \cdot(f \otimes g)\right)= & G^{\Theta} m_{\Theta}(f \otimes g) \\
& -\frac{1}{2} \delta_{G} \theta^{\alpha \beta} \frac{\partial}{\partial \theta^{\alpha \beta}} m_{\Theta}(f \otimes g),
\end{aligned}
$$

where we recall that $\delta_{G} \theta^{\alpha \beta}$ is the Lie derivative of the tensor $\Theta=\theta^{\alpha \beta} \partial_{\alpha} \otimes \partial_{\beta}$ with respect to $G$, see definition (9). For the sake of illustration, this means that for instance 
for dilatations one has

$$
m_{\Theta}\left(\Delta_{\Theta}(D) \cdot(f \otimes g)\right)=D^{\Theta}\left(f \star_{\Theta} g\right)+\theta^{\alpha \beta} \frac{\partial}{\partial \theta^{\alpha \beta}}\left(f \star_{\Theta} g\right) .
$$

Furthermore, observer and twist covariances boil down to

$$
\begin{aligned}
\text { observer: } G^{\Theta} m_{\Theta} & =m_{\Theta} \Delta(G) \\
\text { twist: } G m_{\Theta} & =m_{\Theta} \Delta_{\Theta}(G) .
\end{aligned}
$$

\section{CONCLUSION AND OUTLOOK}

We have investigated noncommutative spacetime transformations from the observer point of view. This regards transformations as coordinate changes under which both the fields and the noncommutativity matrix tensor $\Theta$ transform. The dependence of the Moyal product $f \star_{\Theta} g$ on the spacetime point $x$ and the matrix $\Theta$ leads naturally to introduce an $(x, \Theta)$-space. This is in the spirit of the widespread belief that noncommutativity arises at a certain fundamental length, let it be the Planck length or other. Our main result is that the Moyal product is covariant under linear affine transformations. We obtain explicit expressions for the generators of the Weyl transformations in $(x, \Theta)$-space satisfying two very important properties: they are derivations for the Moyal product and represent the Weyl Lie algebra [18]. This strikingly simplifies the analysis of symmetries of noncommutative field theory actions. The twist approach to noncommutative spacetime transformations has also been revisited and generalized. It is important to remark in connection with the twist formulation, or for that matter, with any formulation of spacetime transformations, that knowledge of a family of generators by itself does not imply invariance of a noncommutative field theory action. This is something that remains to be elucidated.

Possible venues for the future include the understanding of noncommutative gauge transformations in terms of covariance, for a mixing of spacetime coordinates, $\Theta$-variables and gauge degrees of freedom takes place [19]. One may investigate, somewhat along the lines of $[4,20]$, the connection of our approach with the SeibergWitten map [21]. It would also be interesting to study spacetime transformations in which the transformed $x^{\prime \mu}$ depends on both $x^{\mu}$ and $\theta^{\alpha \beta}$. Finally, it is worth extending the covariant approach here to non(anti)commutative superspace [22]. The latter makes sense, since having a representation of the Weyl Lie algebra, it is sensible to ask for supersymmetric extensions in superspace [23].

\section{ACKNOWLEDGMENTS}

The authors are grateful to V. Gayral, G. Marmo and C. Moreno for discussions. JMG-B acknowledges support from MEC, Spain, through a 'Ramón y Cajal' contract. Partial support from CICyT and UCM-CAM, Spain, through grants No. FIS2005-02309, 910770, and from the "Progetto di Ricerca di Interesse Nazionale", Italy, is also acknowledged.

\section{APPENDIX A: ON DERIVATIONS OF THE MOYAL ALGEBRA}

The purpose of this mathematical note is to prove existence of outer derivations for a Moyal algebra. This lies outside the main line of the paper, but stems from the considerations in Sec. II. Being a relevant point that seems to run against standard lore [24], we include it here. A derivation of an associative algebra $\mathcal{F}$ is a linear map $D: \mathcal{F} \rightarrow \mathcal{F}$ satisfying the Leibniz rule

$$
D(a b)=D a b+a D b .
$$

We convene in saying that a derivation $D$ is inner if there is an element $a_{D}$ in $\mathcal{F}$ or in a multiplier algebra of $\mathcal{F}$ such that

$$
D b=a_{D} b-b a_{D}
$$

It is said to be outer if not of this form. For simplicity, consider the Moyal plane $\mathbb{R}^{2}$. We now follow [24] inasmuch as possible. A derivation of the Moyal algebra is entirely determined by its action on $x^{1}, x^{2}$. Let $D_{1}, D_{2}$ be two derivations with

$$
\begin{aligned}
& D_{1} x^{1}=D_{2} x^{1}=f_{1}\left(x^{1}, x^{2}\right) \quad \text { and } \\
& D_{1} x^{2}=D_{2} x^{2}=f_{2}\left(x^{1}, x^{2}\right) .
\end{aligned}
$$

The difference $D_{1}-D_{2}$ is another derivation. It is easily checked to annihilate the plane waves

$$
\left(D_{1}-D_{2}\right) e^{i\left(\alpha x^{1}+i \beta x^{2}\right)}=0 .
$$

It then vanishes. Let now $D$ be an arbitrary derivation. For $D$ to be inner a function $a_{D}$ must exist such that

$$
\begin{aligned}
& {\left[a_{D}, x^{1}\right]_{\star_{\Theta}}=-i \theta \frac{\partial a_{D}}{\partial x^{2}}=f_{1}} \\
& {\left[a_{D}, x^{2}\right]_{\star_{\Theta}}=i \theta \frac{\partial a_{D}}{\partial x^{1}}=f_{2} .}
\end{aligned}
$$

This is the case if and only if the integrability condition $\partial f_{1} / \partial x^{1}+\partial f_{2} / \partial x^{2}=0$ holds. The point is that the derivation $D^{\theta}=x \cdot \partial+2 \theta \frac{\partial}{\partial \theta}$ fulfills $D^{\theta} \theta \neq 0$, so it does not satisfy

$$
\left[D^{\theta} x^{1}, x^{2}\right]_{\star_{\Theta}}=\left[D^{\theta} x^{2}, x^{1}\right]_{\star_{\Theta}},
$$

which is equivalent to the integrability condition. Hence $D^{\theta}$ is outer. Analogous arguments work for our generators $G^{\Theta}$. 
[1] D. Colladay and V. A. Kostelecký, Phys. Rev. D 58, 116002 (1998).

[2] A. Iorio and T. Sýkora, Int. J. Mod. Phys. A 17, 2369 (2002); R. Jackiw and S.-Y. Pi, Phys. Rev. Lett. 88, 111603 (2002); L. Alvarez-Gaumé and M. A. VázquezMozo, Nucl. Phys. B668, 293 (2003).

[3] S. M. Carroll, J. A. Harvey, V. A. Kostelecký, C. D. Lane, and T. Okamoto, Phys. Rev. Lett. 87, 141601 (2001).

[4] A. A. Bichl, J. M. Grimstrup, H. Grosse, E. Kraus, L. Popp, M. Schweda, and R. Wulkenhaar, Eur. Phys. J. C 24, 165 (2002).

[5] C. Gonera, P. Kosinski, P. Maslanka, and S. Giller, Phys. Rev. D 72, 067702 (2005); Phys. Lett. B 622, 192 (2005).

[6] R. Oeckl, Nucl. Phys. B581, 559 (2000).

[7] M. Chaichian, P. P. Kulish, K. Nishijima, and A. Tureanu, Phys. Lett. B 604, 98 (2004).

[8] J. Wess, hep-th/0408080.

[9] P. Matlock, Phys. Rev. D 71, 126007 (2005).

[10] F. Lizzi, S. Vaidya, and P. Vitale, Phys. Rev. D 73, 125020 (2006).

[11] P. Aschieri, C. Blohmann, M. Dimitrijevic, F. Meyer, P. Schupp, and J. Wess, Class. Quant. Grav. 22, 3511 (2005).

[12] D. V. Vassilevich, Mod. Phys. Lett. A 21, 1279 (2006); P. Aschieri, M. Dimitrijevic, F. Meyer, S. Schraml, and J. Wess, hep-th/0603024; J. Zahn, Phys. Rev. D 73, 105005 (2006); M. Chaichian and A. Tureanu, Phys. Lett. B 637, 199 (2006).
[13] M. A. Rieffel, Deformation Quantization for Actions of $\mathbb{R}^{d}$, Memoirs Amer. Math. Soc. Vol. 506 (Providence, RI, USA, 1993).

[14] R. Estrada, J. M. Gracia-Bondía, and J. C. Várilly, J. Math. Phys. (N.Y.) 30, 2789 (1989).

[15] S. Gutt and J. Rawnsley, J. Geom. Phys. 29, 347 (1999).

[16] F. W. Hehl, J. D. McCrea, E. W. Mielke, and Y. Ne'eman, Phys. Rep. 258, 1 (1995).

[17] S. Majid, Foundations of Quantum Group Theory (Cambridge University Press, Cambridge, 1995).

[18] Expressions for the $\theta^{\alpha \beta}$-derivative terms in these generators have been searched for in the past. See M. Chaichian, K. Nishijima, and A. Tureanu, Phys. Lett. B 633, 129 (2006) for an approach to find what we now understand to be $M_{\mu \nu}^{\Theta}-M_{\mu \nu}$.

[19] F. Lizzi, R. Szabo, and A. Zampini, J. High Energy Phys. 08 (2001) 032.

[20] A. Stern and A. Pinzul, Int. J. Mod. Phys. A 20, 5871 (2005).

[21] N. Seiberg and E. Witten, J. High Energy Phys. 09 (1999) 032.

[22] N. Seiberg, J. High Energy Phys. 06 (2003) 010.

[23] For the twist approach, this was first considered in M. Ihl and C. Saemann, J. High Energy Phys. 01 (2006) 065; See also Banerjee, C. Lee and S. Siwach, hep-th/0511205.

[24] M. Dubois-Violette, A. Kriegl, Y. Maeda, and P. W. Michor, Prog. Theor. Phys. Suppl. 144, 54 (2001). 